\title{
Tendência de mortalidade por doenças crônicas não transmissíveis no Centro-0este do Brasil
}

\author{
Lys Bernardes Minasi", Maria Paula Curado*
}

\section{Resumo}

As doenças crônicas não transmissíveis (DCNTs) representam a maior causa de morte no mundo. O objetivo deste estudo foi determinar as tendências de mortalidade por doenças crônicas não transmissíveis no Centro-Oeste do Brasil. Os dados referentes a óbitos foram extraídos do Sistema Informação sobre Mortalidade (SIM), do Ministério da Saúde, disponível no site do Departamento de Informática do SUS (Datasus), para as capitais Goiânia, Cuiabá, Campo Grande e Brasília, no período de 1996 a 2011. As análises foram realizadas por sexo e faixa etária estratificada e agrupada a partir dos 40 anos de idade. Foram registrados, no Centro-Oeste do Brasil, 36.430 óbitos por DCNT no período observado, dos quais, 21.443 indivíduos eram do sexo masculino e 14.987 do feminino. As doenças cardiovasculares foram as mais frequentes, sendo o infarto agudo do miocárdio a principal causa de óbito para ambos os sexos. A tendência de mortalidade para as DCNTs mantiveram-se estáveis no período estudado.

Palavras-chave: Epidemiologia. Fatores de risco. Levantamento epidemiológico.

\section{Introdução}

As Doenças Crônicas Não Transmissíveis (DCNTs) representam a maior causa de morte no mundo, com cerca de 38 milhões de mortes em 2012, dos quais, $46,2 \%$ são por doenças cardiovasculares, $21,7 \%$ por câncer, $10,7 \%$ por doenças respiratórias crônicas e $4,0 \%$ por diabetes, que, tomadas em conjunto, representam $82,6 \%$ dos óbitos por DCNTs. O total de mortes estimado por DCNT para o ano de 2030 é de 52 milhões, segundo dados da World Health Organization (WHO, 2014).

Os principais fatores de risco para as doenças crônicas são hipertensão arterial, tabagismo, hiperglicemia, inatividade física e obesidade. Esses são responsáveis por elevar o risco das doenças crônicas, entre elas, as doenças cardiovasculares e o câncer (BRASIL, 2009; WHO, 2011). Essas doenças são evitáveis

* Mestre em Ciências da Saúde. Área de concentração em Dinâmica do Processo Saúde-Doença Programa de Pós-Graduação em Ciências da Saúde, Universidade Federal de Goiás, Goiânia, Goiás, Brasil. Endereço para correspondência: Avenida Alberto Miguel, n. 140, Setor Campinas, CEP 74510-010, Goiânia, GO. E-mail: lysbernardes@hotmail.com

** Graduada em Medicina pela Universidade Federal de Goiás. Mestre em Medicina pelo Hospital Heliópolis. Doutora em Oncologia. Pesquisadora do Global Burden of Disease. Professora do Programa de Pós-Graduação em Ciências da Saúde da Universidade Federal de Goiás. Professora titular da Fundação Antônio Prudente. E-mail: maria-paula.curado@i-pri.org

$\rightarrow$ http://dx.doi.org/10.5335/rbceh.v13i2.6272

Recebido em: 14/08/2016. Aceito em: 28/03/2017. 
e grande parte das mortes prematuras podem ser reduzidas (WHO, 2008).

Nas últimas décadas, a população brasileira está em franco processo de envelhecimento. A cada ano, 650 mil novos idosos são inclusos na população brasileira, sendo assim, tornam-se necessárias novas estratégias nas políticas públicas de saúde (BRASIL, 2009; INSTITUTO BRASILEIRO DE GEOGRAFIA E ESTATÍSTICA, 2010; VERAS, 2011).

$O$ principal fator de risco para mortalidade por doenças crônicas, no mundo, é a hipertensão arterial, com 13,0\% dos óbitos. Os outros fatores de risco são o tabagismo $(9,0 \%)$, a hiperglicemia $(6,0 \%)$, a inatividade física $(6,0 \%)$ e o excesso de peso e obesidade $(5,0 \%)$ (WHO, 2011; JARDIM et al., 2007). Estima-se que o tabagismo, a dieta pouco saudável e o abuso de bebidas alcoólicas sejam responsáveis por $80,0 \%$ das doenças coronarianas e das cerebrovasculares nos países de baixa e média renda (WHO, 2008).

No Centro-Oeste do Brasil, a população está envelhecendo e o risco para doenças crônicas não transmissíveis é um fato. Existem poucos estudos sobre a mortalidade por DCNT nas capitais dessa região. Portanto, analisar a tendência da mortalidade por doenças crônicas cardiovasculares, diabetes, Alzheimer e o fator de risco obesidade poderá contribuir para identificar o perfil da população.

Analisar a mortalidade pelas doenças crônicas supracitadas nas cidades de Goiânia, Brasília, Cuiabá e Campo Grande, por meio do estudo da tendência de mortalidade na população adulta, poderá trazer informações sobre o perfil das DCNTs nas capitais do Centro-Oeste e poderá auxiliar no planejamento de políticas de saúde pública no controle das doenças crônicas. Nesse contexto, o objetivo geral deste estudo é determinar as taxas e as tendências de mortalidade por doenças crônicas não transmissíveis nas capitais do Centro-Oeste do Brasil, em um período de 16 anos.

\section{Métodos}

Trata-se de um estudo ecológico, retrospectivo, de abordagem quantitativa. A fonte de dados sobre os óbitos por DCNTs foi o Sistema de Informação sobre Mortalidade (SIM), do Ministério da Saúde, disponível no site do Departamento de Informática do SUS. Os dados da população foram extraídos do Datasus, segundo a faixa etária e o sexo, no período de 1996 a 2011 (DATASUS, 2008).

Foram analisados dados da população adulta com idade acima de 40 anos, no período de 1996 a 2011. Os óbitos por DCNT foram selecionados de acordo com o CID-10 e extraídos para as capitais Goiânia, Cuiabá, Campo Grande e Brasília.

A partir dos dados dos óbitos e da população, foram calculados os coeficientes de mortalidade brutos, específicos por idade para cinco faixas etárias (40-49 anos, 50-59 anos, 60-69 anos, 70-79 anos) e agregados de 40 a 79 anos. A seguir, calculou-se o coeficiente de mortalidade padronizado truncado ajustado por idade, tendo como referência a população hipotética proposta por Segi (1960). Na análise de tendência, foram utilizados os valores obtidos pelo cálculo das taxas 
padronizadas por idade. A significância estatística considerada para um nível de significância de $\mathrm{p}<0,05$.

As informações foram inseridas no banco de dados no parâmetro do Microsoft Office Excel ${ }^{\circledR} 2007$ (Microsoft Corporation, EUA). Para as análises das tendências de mortalidade, foi utilizado o modelo de regressão linear, com o auxílio do software Joinpoint Regression Program (Versão 4.0.4, Maio, 2013), disponibilizado pelo National Cancer Institute. Esse modelo de regressão identifica variações significativas nas tendências em um determinado ano.

Para descrever a tendência de cada período, foi obtido o Anual Percent Change (APC), com Intervalo de Confiança (IC) de 95,0\%, com resultado significativo quando $\mathrm{p}<0,05$. A mudança percen- tual anual é significativamente diferente de 0 para alfa $=0,05$.

\section{Resultados}

No período de 1996 a 2011, foram registrados, no Centro-Oeste do Brasil, 36.430 óbitos por DCNT, dos quais, 21.443 eram indivíduos do sexo masculino e 14.987 do sexo feminino (Tabela 1). Tanto para o sexo feminino quanto para o masculino, nas quatro capitais, o infarto agudo do miocárdio foi a causa mais comum de óbito (total de 13.602 óbitos), com maior proporção em Brasília, em homens $37,13 \%$ (3.236 óbitos). A segunda causa de óbito foi o acidente vascular cerebral (AVC), com 7.340 óbitos no período, ou seja, 41,71\% (1.725 óbitos), também em Brasília e no sexo masculino.

Tabela 1 - Proporção de óbitos por DCNTs selecionadas, para os sexos masculino e feminino, no período de 1996 a 2011, nas capitais do Centro-Oeste do Brasil

\begin{tabular}{|c|c|c|c|c|c|c|c|c|c|c|}
\hline & \multicolumn{5}{|c|}{ Sexo feminino } & \multicolumn{5}{|c|}{ Sexo masculino } \\
\hline & Brasília & $\begin{array}{l}\text { Campo } \\
\text { Grande }\end{array}$ & Cuiabá & Goiânia & Total & Brasília & $\begin{array}{l}\text { Campo } \\
\text { Grande }\end{array}$ & Cuiabá & Goiânia & Total \\
\hline & $n(\%)$ & n (\%) & n (\%) & $n(\%)$ & n & $n(\%)$ & n (\%) & $n(\%)$ & $n(\%)$ & n \\
\hline Alzheimer & $125(37,0)$ & $56(16,6)$ & $43(12,7)$ & $114(33,7)$ & 338 & $94(33,9)$ & $61(22,0)$ & $28(10,1)$ & $94(33,9)$ & 277 \\
\hline Angina & $14(31,8)$ & $3(6,8)$ & $6(13,6)$ & $21(47,7)$ & 44 & $26(41,3)$ & $5(7,9)$ & $9(14,3)$ & $23(36,5)$ & 63 \\
\hline Asma & $195(57,9)$ & $56(16,6)$ & $36(10,7)$ & $50(14,8)$ & 337 & $115(60,8)$ & $35(18,5)$ & $14(7,4)$ & $25(13,2)$ & 189 \\
\hline Aterosclerose & $51(53,7)$ & $18(18,9)$ & $3(3,2)$ & $23(24,2)$ & 95 & $66(46,5)$ & $31(21,8)$ & $14(9,9)$ & $31(21,8)$ & 142 \\
\hline AVC & $1436(44,8)$ & $511(15,9)$ & $529(16,5)$ & $728(22,7)$ & 3.204 & $1725(41,7)$ & $734(17,7)$ & $761(18,4)$ & $916(22,1)$ & 4.136 \\
\hline Diabetes & $135(52,7)$ & $29(11,3)$ & $29(11,3)$ & $63(24,6)$ & 256 & $138(54,3)$ & $25(9,8)$ & $18(7,1)$ & $73(28,7)$ & 254 \\
\hline $\begin{array}{l}\text { Doença cardíaca } \\
\text { hipertensiva }\end{array}$ & $708(44,6)$ & $307(19,3)$ & $218(13,7)$ & $354(22,3)$ & 1.587 & $770(45,7)$ & $360(21,4)$ & $263(15,6)$ & $293(17,4)$ & 1.686 \\
\hline $\begin{array}{l}\text { Doença isquêmi- } \\
\text { ca crônica }\end{array}$ & $610(35,3)$ & $287(16,6)$ & $73(4,2)$ & $756(43,8)$ & 1.726 & $1056(34,5)$ & $559(18,3)$ & $147(4,8)$ & $1301(42,5)$ & 3.063 \\
\hline Enfisema & $84(28,8)$ & $57(19,5)$ & $15(5,1)$ & $136(46,6)$ & 292 & $160(28,3)$ & $120(21,2)$ & $41(7,2)$ & $245(43,3)$ & 566 \\
\hline Hipertensão & $547(43,6)$ & $230(18,3)$ & $259(20,7)$ & $218(17,4)$ & 1.254 & $506(45,0)$ & $179(15,9)$ & $274(24,4)$ & $165(14,7)$ & 1.124 \\
\hline $\begin{array}{l}\text { Infarto agudo do } \\
\text { miocárdio }\end{array}$ & $1953(40,0)$ & $1069(21,9)$ & $501(10,3)$ & $1363(27,9)$ & 4.886 & $3236(37,1)$ & $2192(25,1)$ & $958(11,0)$ & $2330(26,7)$ & 8.716 \\
\hline Obesidade & $135(47,2)$ & $63(22,0)$ & $28(9,8)$ & $60(21,0)$ & 286 & $58(35,4)$ & $49(29,9)$ & $19(11,6)$ & $38(23,2)$ & 164 \\
\hline $\begin{array}{l}\text { Outras doenças } \\
\text { isquêmicas }\end{array}$ & $261(38,3)$ & $163(23,9)$ & $72(10,6)$ & $186(27,3)$ & 682 & $416(39,1)$ & $237(22,3)$ & $108(10,2)$ & $302(28,4)$ & 1.063 \\
\hline Total & & & & & 14.987 & & & & & 21.443 \\
\hline
\end{tabular}

Fonte: Datasus (2008). 
A seguir, descreveremos as características epidemiológicas das DCNTs mais comuns e suas tendências nas capitais do Centro-Oeste do Brasil.

\section{Doença de Alzheimer}

No Centro-Oeste do Brasil, ocorreram 616 óbitos por doença de Alzheimer, sendo 277 homens e 338 mulheres. A análise de tendência de mortalidade revelou que, em ambos os sexos, as taxas foram estáveis, com tendência à queda para o sexo feminino (não significativa), nas quatro capitais.

\section{Doenças cardiovasculares}

Ocorreram 33.471 óbitos por doenças cardiovasculares no período observado, sendo 19.993 homens e 13.478 mulheres. A maior taxa de mortalidade foi para o infarto agudo de miocárdio, com 29,23/100.000 em homens e $15,57 / 100.000$ em mulheres.

Observou-se tendência de queda da mortalidade no Centro-Oeste para AVC e para infarto agudo do miocárdio, tanto para o sexo feminino quanto para o masculino. Entretanto, para a doença cardíaca hipertensiva, mostrou-se estável, com menor tendência à queda.

A doença isquêmica crônica mostrou um padrão heterogêneo da tendência de mortalidade, porém, na maioria das vezes, com estabilidade, sem significância estatística.

\section{Obesidade}

Observou-se 450 óbitos por obesidade, sendo 164 para o sexo masculino e 286 para o feminino. As taxas de mortalidade variaram de 0,01 a 1,59/100.000 habitantes nas mulheres e 0,01 a $1,48 / 100.000$ habitantes nos homens. A mortalidade por obesidade manteve-se estável, com tendência de queda (não significativa) para ambos os sexos nas capitais do Centro-Oeste.

\section{Doenças do aparelho respiratório}

Foram registrados 1.384 óbitos por asma e enfisema, sendo 629 em mulheres e 755 em homens. A maior taxa de mortalidade padronizada por asma foi observada no ano de 1998, para o sexo feminino, na cidade de Cuiabá, e, no ano de 1997, em Goiânia, para o sexo masculino (2,13 e 2,15/100.000 habitantes). Para o enfisema, a maior taxa de mortalidade ocorreu em Goiânia, para o sexo masculino e para o feminino $(7,15 \mathrm{e}$ 2,42/100.000 habitantes).

Foi observada queda da mudança percentual anual (APC) para a mortalidade por enfisema, no sexo masculino, em Brasília. Já para o sexo feminino, verificou-se tendência de estabilização da mortalidade, porém não significativa.

Houve tendência de queda da mortalidade por asma em ambos os sexos, mas não significativa.

\section{Diabetes}

Ocorreram 510 óbitos por diabetes, sendo 254 em homens e 256 em mulheres, na proporção de 1 para 1 . A maior taxa de mortalidade foi $1,90 / 100.000$ em Goiânia, para o sexo masculino, e 3,14/100.000 em Cuiabá, para mulheres.

Em Brasília, houve aumento nas tendências da mortalidade por diabetes $(\mathrm{APC}=4.3$; IC95\% -9,6 a 20,3; $\mathrm{p}=0,005)$, 
nos homens, no período de 2000-2011, na faixa etária de 50 a 59 anos.

A análise das tendências de mortalidade para o sexo masculino e DCNT, agregada para faixas etárias de 40 a 79 anos (Tabela 2), revelou que as 10 DCNT mais incidentes no Centro-Oeste do Brasil, mantiveram a mortalidade estável ou crescente, porém sem significância estatística.

Tabela 2 - Tendência da mortalidade por DCNTs, no sexo masculino, idade de 40-79 anos, no período de 1996 a 2011, no Centro-Oeste do Brasil

Continua

\begin{tabular}{|c|c|c|c|c|c|}
\hline \multicolumn{6}{|c|}{ Sexo masculino } \\
\hline DCNT & Capital & Período & APC & $95 \% \mathrm{Cl}$ & $\mathbf{P}$ \\
\hline Alzheimer & $\begin{array}{l}\text { Brasília } \\
\text { Campo Grande } \\
\text { Goiânia } \\
\text { Cuiabá }\end{array}$ & $\begin{array}{l}1996-1998 \\
1998-2001 \\
2001-2011 \\
1996-2011 \\
1996-2011 \\
1996-2011\end{array}$ & $\begin{array}{c}-90,7^{\wedge} \\
283,1^{\wedge} \\
10,6^{\wedge} \\
10,6^{\wedge} \\
25,3^{\wedge} \\
18,4^{\wedge}\end{array}$ & $\begin{array}{c}-97,4 \text { a }-66,9 \\
8,1 \text { a } 1257,5 \\
0,2 \text { a } 22,1 \\
4,9 \text { a } 16,5 \\
12,5 \text { a } 39,5 \\
0,5 \text { a } 39,5\end{array}$ & $\begin{array}{l}0,81 \\
0,81 \\
0,81 \\
0,93 \\
0,03 \\
0,43\end{array}$ \\
\hline Diabetes & $\begin{array}{l}\text { Brasília } \\
\text { Campo Grande } \\
\text { Goiânia } \\
\text { Cuiabá }\end{array}$ & $\begin{array}{l}1996-1999 \\
1999-2011 \\
1996-2011 \\
1996-2000 \\
2000-2011 \\
1996-2011\end{array}$ & $\begin{array}{c}475,0^{\wedge} \\
4,1 \\
26,0 \\
253,2^{\wedge} \\
6,8 \\
38,1^{\wedge}\end{array}$ & $\begin{array}{c}174,7 \text { a } 1103,7 \\
-4,6 \text { a } 13,7 \\
-2,7 \text { a } 63,0 \\
38,7 \text { a } 799,5 \\
-12,5 \text { a } 30,3 \\
11,0 \text { a } 71,9\end{array}$ & $\begin{array}{c}0,99 \\
0,99 \\
0,08 \\
0,003 \\
0,003 \\
0,08\end{array}$ \\
\hline Obesidade & $\begin{array}{l}\text { Brasília } \\
\text { Campo Grande } \\
\text { Goiânia } \\
\text { Cuiabá }\end{array}$ & $\begin{array}{l}1996-2011 \\
1996-1999 \\
1999-2002 \\
2002-2011 \\
1996-1998 \\
1998-2011 \\
1996-2011\end{array}$ & $\begin{array}{c}22,2^{\wedge} \\
-14,5 \\
551,5 \\
15,7 \\
1321,2 \\
-9,8 \\
10,6\end{array}$ & $\begin{array}{c}4,4 \text { a } 43,1 \\
-76,1 \text { a } 205,2 \\
-48,9 \text { a } 206,9 \\
-8,3 \text { a } 45,9 \\
-61,3 \text { a } 52042,9 \\
-25,3 \text { a } 9,0 \\
-12,3 \text { a } 39,4\end{array}$ & $\begin{array}{c}0,22 \\
0,009 \\
0,009 \\
0,009 \\
0,009 \\
0,009 \\
0,04\end{array}$ \\
\hline Asma & $\begin{array}{l}\text { Brasília } \\
\text { Campo Grande } \\
\text { Goiânia } \\
\text { Cuiabá }\end{array}$ & $\begin{array}{l}1996-2011 \\
1996-2011 \\
1996-2011 \\
1996-2011\end{array}$ & $\begin{array}{c}-9,1^{\wedge} \\
-15,0^{\wedge} \\
-11,6 \\
13,6\end{array}$ & $\begin{array}{c}-12,8 \text { a }-5,3 \\
-24,8 \text { a }-3,9 \\
-27,4 \text { a } 7,8 \\
-12,3 \text { a } 47,1\end{array}$ & $\begin{array}{l}0,44 \\
0,25 \\
0,64 \\
0,95\end{array}$ \\
\hline Enfisema & $\begin{array}{l}\text { Brasília } \\
\text { Campo Grande } \\
\text { Goiânia } \\
\text { Cuiabá }\end{array}$ & $\begin{array}{l}1996-2011 \\
1996-2011 \\
1996-2011 \\
1996-2011\end{array}$ & $\begin{array}{c}-5,6^{\wedge} \\
-8,4^{\wedge} \\
-10,1^{\wedge} \\
0,4^{\wedge}\end{array}$ & $\begin{array}{c}-9,1 \text { a }-2,0 \\
-12,8 \text { a }-3,8 \\
-13,5 \text { a }-6,5 \\
-5,6 \text { a } 6,9\end{array}$ & $\begin{array}{l}0,14 \\
0,30 \\
0,16 \\
0,15\end{array}$ \\
\hline Aterosclerose & $\begin{array}{l}\text { Brasília } \\
\text { Campo Grande } \\
\text { Goiânia } \\
\text { Cuiabá }\end{array}$ & $\begin{array}{l}1996-2011 \\
1996-2011 \\
1996-2011 \\
1996-2011 \\
\end{array}$ & $\begin{array}{c}-10,6^{\wedge} \\
-12,8^{\wedge} \\
-22,7^{\wedge} \\
-2,5\end{array}$ & $\begin{array}{c}-16,4 \text { a }-4,5 \\
-22,6 \text { a }-1,8 \\
-32,9 \text { a }-10,9 \\
-25,6 \text { a } 27,7\end{array}$ & $\begin{array}{l}0,77 \\
0,54 \\
0,09 \\
0,52\end{array}$ \\
\hline Angina & $\begin{array}{l}\text { Brasília } \\
\text { Campo Grande } \\
\text { Goiânia } \\
\text { Cuiabá }\end{array}$ & $\begin{array}{l}1996-2011 \\
1996-2011 \\
1996-2011 \\
1996-2011\end{array}$ & $\begin{array}{r}1,3 \\
-5,1 \\
2,2 \\
6,7\end{array}$ & $\begin{array}{l}-11,1 \text { a } 15,4 \\
-25,0 \text { a } 20,1 \\
-16,8 \text { a } 25,4 \\
-18,4 \text { a } 39,6\end{array}$ & $\begin{array}{l}0,24 \\
0,14 \\
0,93 \\
0,64\end{array}$ \\
\hline$\overline{A V C}$ & $\begin{array}{l}\text { Brasília } \\
\text { Campo Grande } \\
\text { Goiânia } \\
\text { Cuiabá }\end{array}$ & $\begin{array}{l}1996-2011 \\
1996-2011 \\
1996-2011 \\
1996-2011\end{array}$ & $\begin{array}{l}-6,1^{\wedge} \\
-7,4^{\wedge} \\
-5,3^{\wedge} \\
-9,7^{\wedge}\end{array}$ & $\begin{array}{c}-6,1^{\wedge} \text { a }-4,8 \\
-9,6 \text { a }-5,3 \\
-7,7 \text { a }-2,8 \\
-11,9 \text { a }-7,4\end{array}$ & $\begin{array}{l}0,66 \\
0,48 \\
0,16 \\
0,37\end{array}$ \\
\hline $\begin{array}{l}\text { Doença cardíaca } \\
\text { hipertensiva }\end{array}$ & $\begin{array}{l}\text { Brasília } \\
\text { Campo Grande } \\
\text { Goiânia } \\
\text { Cuiabá }\end{array}$ & $\begin{array}{l}1996-2011 \\
1996-2011 \\
1996-2011 \\
1996-2011\end{array}$ & $\begin{array}{c}-0,2 \\
8,5^{\wedge} \\
-0,2 \\
10,7^{\wedge}\end{array}$ & $\begin{array}{l}-3,5 \text { a } 3,2 \\
5,1 \text { a } 12,0 \\
-3,5 \text { a } 3,2 \\
4,7 \text { a } 17,0\end{array}$ & $\begin{array}{l}0,13 \\
0,47 \\
0,13 \\
0,04\end{array}$ \\
\hline
\end{tabular}


Conclusão

\begin{tabular}{l|l|c|c|c|c}
\hline Doença isquêmica & Brasília & $1996-2001$ & $-9,0^{\wedge}$ & $-10,5$ a $-7,4$ & 0,35 \\
crônica do coração & Campo Grande & $1996-2001$ & $28,1^{\wedge}$ & 4,7 a 56,7 & 0,01 \\
& & $2001-2011$ & $-5,4$ & $-11,9$ a 1,4 & 0,01 \\
& Goiânia & $1996-2001$ & $-0,9$ & $-11,6$ a 11,2 & 0,44 \\
& & $2001-2004$ & 46,3 & $-12,5$ a 144,4 & 0,44 \\
& & $2004-2011$ & $-8,8^{\wedge}$ & $-14,8$ a $-2,3$ & 0,44 \\
\hline Hipertensão & Cuiabá & $1996-2001$ & 1,9 & $-4,4$ a 8,7 & 0,43 \\
arterial & Brasília & $1996-1998$ & 68,4 & $-5,3$ a 199,6 & 0,58 \\
& Campo Grande & $1998-2011$ & $-2,2$ & $-5,1$ a 0,8 & 0,58 \\
& & $1996-2000$ & $-24,5$ & $-51,1$ a 16,5 & 0,02 \\
& Goiânia & $2000-2011$ & $10,3^{\wedge}$ & 0,5 a 21,0 & 0,02 \\
\hline Infarto agudo do & Cuiabá & $1996-2011$ & 2,8 & $-2,5$ a 8,4 & 0,85 \\
miocárdio & Brasília & $2005-2011$ & $-19,8$ & $-2,3$ a 16,8 & 0,007 \\
& Campo Grande & $1996-2011$ & $-19,4 \wedge$ & $-31,7$ a $-4,9$ & 0,007 \\
\hline Outras doenças & Goiânia & $1996-2011$ & 0,8 & $-3,0$ a 0,2 & 0,06 \\
isquêmicas & Cuiabá & $1996-2011$ & $3,6 \wedge$ & $-0,4$ a 1,9 & 0,28 \\
agudas do & Camília & $1996-2011$ & $-0,9$ & 1,1 a 6,1 & 0,13 \\
coração & Goiâno Grande & $1996-2011$ & $-7,7^{\wedge}$ & $-2,3$ a 0,6 & 0,11 \\
\hline
\end{tabular}

Fonte: Datasus (2008).

Nota: ^ A Mudança Percentual Anual (APC) é significativamente diferente de zero para alfa = 0,05.

$\wedge$ The Annual Percentage Change (APC) is significantly different from zero for alpha $=0.05$.

As doenças de Alzheimer, diabetes e obesidade mostraram tendência de aumento não significativo, já as doenças respiratórias e cardiovasculares apresentaram tendência de estabilidade.

No sexo feminino, a tendência de mortalidade agregada para idade de 40 a 79 anos (Tabela 3 ) revelou tendência de aumento para Alzheimer, diabetes e obesidade. As doenças cardiovasculares apresentaram tendência de queda, porém não significativa, nas capitais do Centro-Oeste (Tabela 3). 
Tabela 3 - Tendência da mortalidade por DCNTs, no sexo feminino, para idade de 40-79 anos, no período de 1996 a 2011, no Centro-Oeste do Brasil

Continua

\begin{tabular}{|c|c|c|c|c|c|}
\hline \multicolumn{6}{|c|}{ Sexo feminino } \\
\hline DCNT & Capital & Período & APC & $95 \% \mathrm{Cl}$ & $\mathrm{P}$ \\
\hline Alzheimer & $\begin{array}{l}\text { Brasília } \\
\text { Campo Grande } \\
\text { Goiânia } \\
\text { Cuiabá }\end{array}$ & $\begin{array}{l}1996-2011 \\
1996-2011 \\
1996-2011 \\
1996-1999 \\
1999-2011\end{array}$ & $\begin{array}{c}18,7^{\wedge} \\
48,3^{\wedge} \\
11,9^{\wedge} \\
445,5^{\wedge} \\
12,5^{\wedge}\end{array}$ & $\begin{array}{c}5,9 \text { a } 33,1 \\
25,5 \text { a } 75,2 \\
4,9 \text { a } 19,4 \\
189,0 \text { a } 929,5 \\
4,4 \text { a } 21,3\end{array}$ & $\begin{array}{c}0,06 \\
0,84 \\
0,48 \\
0,004 \\
0,004\end{array}$ \\
\hline Diabetes & $\begin{array}{l}\text { Brasília } \\
\text { Campo Grande } \\
\text { Goiânia } \\
\text { Cuiabá }\end{array}$ & $\begin{array}{c}1996-1998 \\
1998-2011 \\
1996-2011 \\
1996-1999 \\
1999-2011 \\
1996-2011\end{array}$ & $\begin{array}{c}616,4^{\wedge} \\
4,9 \\
0,9 \\
328,2^{\wedge} \\
5,9 \\
4,6\end{array}$ & $\begin{array}{c}103,9 \text { a } 2417,4 \\
-1,8 \text { a } 12,0 \\
-19,9 \text { a } 27,0 \\
74,7 \text { a } 949,7 \\
-4,8 \text { a } 17,7 \\
-21,5 \text { a } 39,3\end{array}$ & $\begin{array}{l}0,98 \\
0,98 \\
0,54 \\
0,50 \\
0,50 \\
0,38\end{array}$ \\
\hline Obesidade & $\begin{array}{l}\text { Brasília } \\
\text { Campo Grande } \\
\text { Goiânia } \\
\text { Cuiabá }\end{array}$ & $\begin{array}{l}1996-2001 \\
2001-2011 \\
1996-2011 \\
1996-2011 \\
1996-2011\end{array}$ & $\begin{array}{c}169,8^{\wedge} \\
8,5^{\wedge} \\
45,4^{\wedge} \\
16,0^{\wedge} \\
40,5^{\wedge}\end{array}$ & $\begin{array}{c}82,3 \text { a } 299,4 \\
-5,4 \text { a } 24,3 \\
24,3 \text { a } 70,0 \\
3,3 \text { a } 30,1 \\
17,0 \text { a } 68,6\end{array}$ & $\begin{array}{l}0,35 \\
0,35 \\
0,41 \\
0,20 \\
0,41\end{array}$ \\
\hline Asma & $\begin{array}{l}\text { Brasília } \\
\text { Campo Grande } \\
\text { Goiânia } \\
\text { Cuiabá }\end{array}$ & $\begin{array}{l}1996-2011 \\
1996-2011 \\
1996-2011 \\
1996-2011\end{array}$ & $\begin{array}{c}-6,2 \wedge \\
-12,2 \\
-8,5 \\
6,7\end{array}$ & $\begin{array}{l}-9,0 \text { a }-3,3 \\
-27,4 \text { a } 6,1 \\
-20,1 \text { a } 4,7 \\
-8,2 \text { a } 24,0\end{array}$ & $\begin{array}{l}0,05 \\
0,39 \\
0,35 \\
0,03\end{array}$ \\
\hline Enfisema & $\begin{array}{l}\text { Brasília } \\
\text { Campo Grande } \\
\text { Goiânia } \\
\text { Cuiabá }\end{array}$ & $\begin{array}{l}1996-2011 \\
1996-2011 \\
1996-2006 \\
2006-2011 \\
1996-2011\end{array}$ & $\begin{array}{c}-8,4^{\wedge} \\
-12,3 \\
-1,0 \\
-23,0^{\wedge} \\
-12,3\end{array}$ & $\begin{array}{c}-13,6 \text { a }-2,9 \\
-31,5 \text { a } 12,4 \\
-8,1 \text { a } 6,6 \\
-37,8 \text { a }-4,6 \\
-31,5 \text { a } 12,4\end{array}$ & $\begin{array}{l}0,79 \\
0,82 \\
0,06 \\
0,06 \\
0,82\end{array}$ \\
\hline Angina & $\begin{array}{l}\text { Brasília } \\
\text { Campo Grande } \\
\text { Goiânia } \\
\text { Cuiabá }\end{array}$ & $\begin{array}{l}1996-2011 \\
1996-2011 \\
1996-2011 \\
1996-2011\end{array}$ & $\begin{array}{c}1,2 \\
20,9 \wedge \\
-0,4 \\
-2,6\end{array}$ & $\begin{array}{c}-15,6 \text { a } 21,5 \\
3,4 \text { a } 41,4 \\
-19,5 \text { a } 23,3 \\
-25,2 \text { a } 26,9\end{array}$ & $\begin{array}{l}0,43 \\
0,30 \\
0,20 \\
0,21\end{array}$ \\
\hline Aterosclerose & $\begin{array}{l}\text { Brasília } \\
\text { Campo Grande } \\
\text { Goiânia } \\
\text { Cuiabá }\end{array}$ & $\begin{array}{l}1996-2011 \\
1996-2011 \\
1996-2011 \\
1996-2011\end{array}$ & $\begin{array}{c}-14,8^{\wedge} \\
-8,6 \\
-16,5 \\
7,8\end{array}$ & $\begin{array}{c}-20,1 \text { a }-9,1 \\
-25,5 \text { a } 12,0 \\
-31,8 \text { a } 2,3 \\
-9,5 \text { a } 28,4\end{array}$ & $\begin{array}{l}0,13 \\
0,77 \\
0,06 \\
0,63\end{array}$ \\
\hline$\overline{A V C}$ & $\begin{array}{l}\text { Brasília } \\
\text { Campo Grande } \\
\text { Goiânia } \\
\text { Cuiabá }\end{array}$ & $\begin{array}{l}1996-1998 \\
1998-2001 \\
2001-2006 \\
2006-2011 \\
1996-2011 \\
1996-2001 \\
2001-2011 \\
1996-2011\end{array}$ & $\begin{array}{c}19,8 \\
-16,2 \\
-3,1 \\
-9,1^{\wedge} \\
-7,6^{\wedge} \\
-15,6^{\wedge} \\
-1,3 \\
-9,7^{\wedge}\end{array}$ & $\begin{array}{c}-4,5 \text { a } 50,2 \\
-33,2 \text { a } 5,0 \\
-9,8 \text { a } 4,1 \\
-13,6 \text { a }-4,4 \\
-9,8 \text { a }-5,4 \\
-20,8 \text { a }-10,1 \\
-3,5 \text { a } 0,9 \\
-11,9 \text { a }-7,4\end{array}$ & $\begin{array}{c}0,002 \\
0,002 \\
0,002 \\
0,002 \\
0,22 \\
0,84 \\
0,84 \\
0,37\end{array}$ \\
\hline \multirow[t]{2}{*}{$\begin{array}{l}\text { Doença cardíaca } \\
\text { hipertensiva }\end{array}$} & $\begin{array}{l}\text { Brasília } \\
\text { Campo Grande } \\
\text { Goiânia }\end{array}$ & $\begin{array}{l}1996-2011 \\
1996-2011 \\
1996-1998 \\
1998-2003 \\
2003-2006\end{array}$ & $\begin{array}{c}7,3^{\wedge} \\
7,3^{\wedge} \\
-30,0^{\wedge} \\
19,1^{\wedge} \\
-34,8^{\wedge}\end{array}$ & $\begin{array}{c}2,5 \text { a } 12,3 \\
2,5 \text { a } 12,3 \\
-49,5 \text { a }-3,1 \\
7,4 \text { a } 32,0 \\
-52,9 \text { a }-9,6\end{array}$ & $\begin{array}{c}0,11 \\
0,0006 \\
0,0006 \\
0,0006\end{array}$ \\
\hline & Cuiabá & $\begin{array}{l}2006-2011 \\
1996-2011\end{array}$ & $\begin{array}{c}16,1^{\wedge} \\
9,8^{\wedge}\end{array}$ & $\begin{array}{l}8,0 \text { a } 24,9 \\
5,2 \text { a } 14,6\end{array}$ & $\begin{array}{c}0,0006 \\
0,99\end{array}$ \\
\hline
\end{tabular}


Conclusão

\begin{tabular}{l|l|c|c|cc|c} 
Doença isquêmica & Brasília & $1996-2011$ & $-8,9 \wedge$ & $-11,0$ a $-6,7$ & 0,14 \\
crônica do coração & Campo Grande & $1996-2001$ & $40,7 \wedge$ & 4,1 a 90,2 & 0,01 \\
& & $2001-2011$ & $-7,6$ & $-16,8$ a 2,6 & 0,01 \\
& Goiânia & $1996-2006$ & $14,0^{\wedge}$ & 8,0 a 20,3 & 0,15 \\
& & $2006-2011$ & $-16,9 \wedge$ & $-28,8$ a $-3,0$ & 0,15 \\
Hipertensão arterial & Cuiabá & $1996-1998$ & $947,9 \wedge$ & 210,7 a 3434,0 & 0,96 \\
& & $1998-2011$ & $-1,9$ & $-7,9$ a 4,6 & 0,96 \\
& Brasília & $1996-2001$ & 12,3 & $-0,7$ a 27,0 & 0,01 \\
Infarto agudo do & Campo Grande & $2001-2011$ & $-4,0$ & $-8,0$ a 0,2 & 0,01 \\
miocárdio & Goiânia & $1996-2011$ & $-2,8$ & $-5,7$ a 0,2 & 0,76 \\
& Cuiabá & $1996-2011$ & $-2,0$ & $-6,2$ a 2,5 & 0,46 \\
Outras doenças & Brasília & $1996-2011$ & $-3,1$ & $-8,0$ a 1,9 & 0,07 \\
agudas do coração & Campo Grande & $1996-2011$ & $-1,9 \wedge$ & $-3,0$ a $-0,8$ & 0,59 \\
& Goiânia & $1996-2011$ & $-1,5$ & $-3,5$ a 0,4 & 0,06 \\
& Cuiabá & $1996-2011$ & $-0,5$ & $-2,0$ a 1,0 & 0,64 \\
\hline
\end{tabular}

Fonte: Datasus (2008).

Nota: ^ A Mudança Percentual Anual (APC) é significativamente diferente de zero para alfa = 0,05.

$\wedge$ The Annual Percentage Change (APC) is significantly different from zero for alpha $=$

\section{Discussão}

O Brasil é dividido em cinco grandes regiões (Norte, Nordeste, Centro-Oeste, Sudeste e Sul), abrangendo 26 estados e o Distrito Federal, que apresentam uma heterogeneidade demográfica, social e econômica, que reflete diferentes padrões de mortalidade e de morbidade por DCNT. Com isso, exige-se respostas do setor público que envolvam os gestores locais e que sejam adequadas à realidade encontrada em cada estado (BRASIL, 2005).

Em um período de 16 anos (19962011), observou-se 36.430 óbitos por doenças crônicas no Centro-Oeste, em média, 2.295 óbitos por ano (excluindo-se o câncer), sendo que o sexo masculino foi o mais acometido. Segundo Alves e Morais Neto (2015), é importante o conhecimento detalhado do perfil das DNCT para promover a prevenção, assim como o diagnóstico precoce, evitando, assim, as sequelas do tratamento.

Segundo a Organização Mundial de Saúde, populações com menos de 60 anos de idade representam 13,0\% de mortes por DCNT nos países de alta renda e $41,0 \%$ nos de baixa renda (WHO, 2011). Um estudo realizado por Rocha-Brischiliari et al. (2014), com 453 adultos do município de Maringá no estado do Paraná, observou que as DCNT foram mais prevalentes nos idosos e nos indivíduos de baixa escolaridade (ROCHA-BRISCHILIARI et al., 2014). As capitais e os municípios do Brasil têm grandes diferenças sociais, portanto, para entender a mortalidade por doenças crônicas não transmissíveis, é necessário também observar as diferenças socioe- 
conômicas e compreender o processo de envelhecimento da população.

\section{Doença de Alzheimer}

Segundo Teixeira et al. (2015), a tendência de mortalidade por doença de Alzheimer está aumentando consistentemente em ambos os sexos nas faixas etárias acima de 60 anos, e esse aumento não está relacionado apenas à melhora da capacidade diagnóstica, mas, também, à maior longevidade da população.

No presente estudo, a doença de Alzheimer se mostrou estável e com tendência à queda. Porém, a população brasileira está envelhecendo e, com o avançar dos anos, esse resultado poderá ser modificado. Observou-se maior frequência dos óbitos no sexo feminino. $\mathrm{Na}$ França, as mortes por Doença de Alzheimer ou outras demências correspondem a $10,3 \%$ de todas as causas de óbitos nos indivíduos que com idade maior ou igual a 60 anos, sendo mais frequente nas mulheres (BROSSELIN; DUPORT; BLOCH, 2010).

\section{Doenças cardiovasculares}

As doenças do aparelho circulatório representam as maiores causas de mortalidade dentre as doenças crônicas. Observamos que o sexo masculino foi o que obteve a maior frequência de óbitos. Em estudo realizado no Paraná, a maioria dos óbitos por doenças cardiovasculares foram do sexo masculino, com 57,0\%, na faixa etária de 45 a 64 anos (FURUKAWA; SANTO; MATHIAS, 2011), resultado que corrobora os resultados deste trabalho.

Segundo Souza et al. (2012), a doença isquêmica do coração foi a principal causa de óbitos para as doenças cardiovasculares nas Américas. A doença isquêmica crônica diminuiu $24,7 \%$ e a cerebrovascular $21,1 \%$. Segundo Baena et al. (2012), o infarto agudo do miocárdio foi a primeira causa de óbito dentre as doenças crônicas não transmissíveis, sendo observada uma redução na taxa de óbito a partir dos 50 anos de idade em Curitiba, Paraná (BAENA et al., 2012). No Centro-Oeste do Brasil, observa-se uma queda da mortalidade, embora sem significância estatística, o que caracteriza um achado positivo que pode ser atribuído ao diagnóstico precoce dos fatores de risco associados ao infarto agudo do miocárdio e a uma população mais informada.

Um estudo nas regiões Centro-Oeste, Sul e Sudeste demonstrou que há uma tendência decrescente das taxas de mortalidade por doença cardiovascular na faixa etária de 30 a 39 anos, para ambos os sexos (VILLELA; GOMES, MELÉNDEZ, 2014).

Os fatores de risco como hipertensão, tabagismo, diabetes, inatividade física, obesidade e hábitos alimentares inadequados contribuem para o aumento da incidência das doenças crônicas (DOBE, 2012; PEIXOTO et al., 2008). O diagnóstico precoce, a eficácia do tratamento e a conscientização de hábitos de vida saudáveis podem diminuir a incidência e, consequentemente, a mortalidade das doenças crônicas. 


\section{Obesidade}

A obesidade é uma preocupação dos países em desenvolvimento. Destaca-se que a prevalência da obesidade muda de acordo com grupo etário, grupo racial e étnico para homens e mulheres (FLEGAL et al., 2010). No presente estudo, observamos que a mortalidade por obesidade manteve-se estável, com tendência a aumento. Portanto, existe a necessidade de programas educativos eficientes para reduzir a obesidade, como por meio da atividade física e de dieta orientadas por profissionais de cada área.

\section{Diabetes}

Nas décadas de 1980 e 1990, Lessa (2004) observou um crescimento na tendência da mortalidade por diabetes no Brasil, sendo em maior magnitude para as mulheres. ${ }^{22}$ Entretanto, um estudo desenvolvido no Brasil apontou uma tendência estacionária, entre os anos de 1991 a 2010, em ambos os sexos, nas taxas padronizadas de mortalidade (STEVENS; SCHMIDT; DUNCAN, 2012). Segundo Roglic e Unwin (2010), o número de mortes por diabetes apresentou-se em maior proporção em mulheres, sendo que para ambos os sexos correspondeu a $6,8 \%$ da mortalidade por todas as causas. Mattos et al. (2012) observaram tendência de aumento da mortalidade por diabetes, de 1980 a 2007, para a região Centro-Oeste brasileira. No presente estudo, a frequência de óbitos foi maior para o sexo feminino. Observamos, ainda, tendência de estabilidade e de au- mento da mortalidade no Centro-Oeste do Brasil, com variações de acordo com faixa etária e região.

\section{Doenças respiratórias}

Segundo Schmidt et al. (2011), foi observada queda da mortalidade para asma (SCHMIDT, 2011) no Brasil. A maior taxa de mortalidade foi observada na população acima de 65 anos (CAMPOS, 2004). As doenças respiratórias crônicas, no presente estudo, mostraram-se com tendência de estabilidade para o enfisema e tendência de queda para asma, em ambos os sexos. Um dos fatores associados é o tabagismo, cuja prevalência no Centro-Oeste é de 16,9\% (INSTITUTO BRASILEIRO DE GEOGRAFIA E ESTATÍSTICA, 2008). Portanto, intensificar as medidas contra o tabagismo poderá diminuir a incidência de enfisema. Já quanto à asma, é importante destacar que a urbanização e a modernização acarretam a poluição do meio ambiente, o que pode contribuir para o aumento dos casos da doença (COOPER et al., 2009; ISER et al. , 2012; ARBEX et al., 2012). A tendência de mortalidade no Centro-Oeste para doenças respiratórias foi heterogênea. Portanto, é importante observar o padrão dos fatores de risco em cada capital estudada para a adoção de políticas de saúde eficazes.

Malta e Silva (2014) mostraram um declínio médio de $2,5 \%$ ao ano para causas de óbito mais frequentes (doenças cardiovasculares, neoplasias, respiratórias e diabetes) em todas as regiões do Brasil. No presente estudo, observamos 
tendência de estabilidade da mortalidade para praticamente todas as faixas etárias analisadas e em ambos os sexos.

Nos dados secundários analisados, provenientes do Datasus, existem subregistros e causas mal definidas, que não foram computadas nesta análise. $\mathrm{O}$ preenchimento inadequado da declaração de óbito por profissionais da classe médica é uma limitação deste estudo, sendo que, em algumas regiões do Brasil, as causas mal definidas podem chegar a até 20,0\% (SANTO, 2008; MENDONÇA; DRUMOND; CARDOSO, 2010).

\section{Conclusão}

$\mathrm{O}$ presente estudo identificou estabilidade da mortalidade por DCNT nas capitais do Centro-Oeste do Brasil, fato que reflete ações governamentais de saúde pública que poderão reduzir o risco dessa população em médio e longo prazo.

Os resultados desta análise evidenciam a necessidade de ações e investimentos para enfrentar as DCNTs, pois, com isso, os novos estudos de mortalidade poderão demonstrar a queda da mortalidade por doenças crônicas.

\section{Trends in mortality from chronic diseases in Midwest of Brazil}

\section{Abstract}

The Chronic Noncommunicable Diseases (NCD) are the leading cause of death worldwide. The objective was to determine trends in mortality from chronic diseases in the Midwest of Brazil. Deaths were extracted from the System Information (SIM) of the Ministry of the available Health at the SUS Department of Information website (Datasus) to the cities of Goiânia, Cuiabá, Campo Grande and Brasilia, from 1996 to 2011. Analyses were performed by sex and stratified and clustered age from 40 years old. Were recorded, in the Midwest of Brazil, 36.430 deaths from 1996 to 2011 of these 21.443 deaths in men and 14.987 in women. Cardiovascular diseases were the most common being acute myocardial infarction the leading cause of death for both sexes. The trend of mortality for NCDs remained stable during the study period.

Keywords: Risk factors. Epidemiology. Health surveys.

\section{Referências}

ALVES, C. G.; MORAIS NETO, O. L. Tendência da mortalidade prematura por doenças crônicas não transmissíveis nas unidades federadas brasileiras. Ciência \& Saúde Coletiva, Rio de Janeiro (RJ), v. 20, n. 3, p. 641-654, mar. 2015.

ARBEX, M. A. et al. A poluição do ar e o sistema respiratório. Jornal Brasileiro de Pneumologia, São Paulo (SP), v. 38, n. 5, p. 643-655, set./out. 2012.

BAENA, C. P. et al. Tendência de mortalidade por infarto agudo do miocárdio em Curitiba (PR) no período de 1998 a 2009. Arquivos Brasileiros de Cardiologia, São Paulo (SP), v. 98 , n. 3, p. 211-217, mar. 2012.

BRASIL. Manual de instruções para o preenchimento da declaração de óbito. Brasília: [s.n.], 2011. 
Ministério da Saúde. A vigilância e a prevenção das doenças crônicas não transmissíveis: DCNT no contexto do Sistema Único de Saúde brasileiro. Brasília: Ministério da Saúde, 2005.

Ministério da Saúde. Saúde Brasil 2008: 20 anos de Sistema Único de Saúde (SUS) no Brasil. Brasília: Ministério da Saúde, 2009. Disponível em: <http://portal. saude.gov.br/portal/arquivos/pdf/saude_brasil_2008_web_20_11.pdf>. Acesso em: 21 out. 2012.

BROSSELIN, P.; DUPORT, N.; BLOCH, J. Mortality with Alzheimer's disease and dementia in France, 2006. Revue d'Épidemiologie et de Santé Publique, Paris, v. 58, n. 4, p. 269-276, ago. 2010.

CAMPOS, H. S. Asma e DPOC: vida e morte. Boletim de Pneumologia Sanitária, Rio de Janeiro (RJ), v. 12, n. 01, p. 37-53, abr. 2004.

COOPER, P. S. et al. Asthma in Latin America: a public heath challenge and research opportunity. Allergy, Quito (EC), v. 64, n. 1, p. 5-17, jan. 2009.

DATASUS. Ministério da Saúde. CID 10: Classificação Estatística Internacional de Doenças e Problemas Relacionados à Saúde. Brasília: Ministério da Saúde, 2008. Disponível em: <http://www.datasus.gov.br/cid10/ V2008/cid10.htm>. Acesso em: 21 out. 2013.

DOBE, M. Health promotion for prevention and control of non-communicable diseases: unfinished agenda. Indian Journal of Public Health, Kolkata (IND), v. 56, n. 3, p. 180-186, Jan. 2012.

FLEGAL, K. M. et al. Prevalence and trends in obesity among US adults, 1999-2008. The Journal of the American Medical Association, v. 303, n. 3, p. 235-241, Feb. 2010.

FURUKAWA, T. S.; SANTO, A. H.; MATHIAS, T. A. F. Causas múltiplas de morte relacionadas às doenças cerebrovasculares no Estado do Paraná. Revista Brasileira de Epidemiologia, São Paulo (SP), v. 14, n. 2, p. 231-239, nov. 2011.
INSTITUTO BRASILEIRO DE GEOGRAFIA E ESTATÍSTICA. Pesquisa nacional por amostra de domicílios: tabagismo. Rio de Janeiro: IBGE, 2008.

. Síntese de Indicadores Sociais: uma análise das condições de vida da população brasileira. Rio de Janeiro: IBGE, 2010. Disponível em: <http://www.ibge.gov.br/ home/estatistica/populacao/condicaodevida/ indicadoresminimos/sinteseindicsociais2010/ SIS_2010.pdf>. Acesso em: 25 out. 2012.

ISER, B. P. M. et al. Prevalência de fatores de risco e proteção para doenças crônicas nas capitais do Brasil - principais resultados do Vigitel 2010. Ciência \& Saúde Coletiva, Rio de Janeiro (RJ), v. 17, n. 9, p. 2343-2356, set. 2012.

JARDIM, P. C. B. et al. Hipertensão arterial e alguns fatores de risco em uma capital brasileira. Arquivos Brasileiros de Cardiologia, Rio de Janeiro (RJ), v. 88, n. 4, p. 452-457, out. 2007.

LESSA, I. Doenças crônicas não-transmissíveis no Brasil: um desafio para a complexa tarefa da vigilância. Ciência \& Saúde Coleti$v a$, Rio de Janeiro (RJ), v. 9, n. 4, p. 931-943, out./dez. 2004.

MALTA, D. C.; SILVA, J. B. Plano de ações estratégicas para o enfrentamento das doenças crônicas não transmissíveis no Brasil após três aos de implantação, 2011-2013. Epidemiologia e Serviços de Saúde, Brasília (BSB), v. 23, n. 3, p. 389-395, jul./set. 2014.

MATTOS, P. E. et al. Tendência da mortalidade por diabetes melito em capitais brasileiras, 1980-2007. Arquivos Brasileiros de Endocrinologia \& Metabologia, São Paulo (SP), v. 56, n. 1, p. 39-46, fev. 2012.

MENDONÇA, F. M.; DRUMOND, E.; CARDOSO, A. M. P. Problemas no preenchimento da declaração de óbito: estudo exploratório. Revista Brasileira de Estudos de População, São Paulo (SP), v. 27, n. 2, p. 285-295, jul./ dez. 2010. 
PEIXOTO, M. R. G. et al. Monitoramento por entrevistas telefônicas de fatores de risco para doenças crônicas: experiência de Goiânia, Goiás, Brasil. Caderno de Saúde Pública, Rio de Janeiro (RJ), v. 24, n. 6, p. 1323-1333, jan./jun. 2008.

ROCHA-BRISCHILIARI, S. C. et al. Doenças Crônicas não Transmissíveis e Associação com Fatores de Risco. Revista Brasileira de Cardiologia, Rio de Janeiro (RJ), v. 27, n. 1, p. 531-538, jan./fev. 2014.

ROGLIC, G.; UNWIN, N. Mortality attributable to diabetes: estimates for the year 2010. Diabetes Research and Clinical Practice, v. 87, n. 1, p. 15-19, Jan. 2010.

SANTO, A. H. Causas mal definidas de mortes e óbitos sem assistência. Revista da Associação Médica Brasileira, São Paulo (SP), v. 54, n. 1, p. 23-28, jan./fev. 2008.

SCHMITCH, M. I. et al. Chronic noncommunicable diseases in Brazil: burden and current challenges. The Lancet, v. 377, n. 9781, p. 1949-1961, June. 2011.

SEGI, M. Cancer Mortality for Selected Sites in 24 Countries (1950-57). Sendai: Department of Public Health, Tohoku University School of Medicine - Japan, 1960.

SOUZA, M. F. M. et al. Cardiovascular disease mortality in the Americas: current trends and disparities. Heart, v. 98, n. 16, p. 1207-1212, 2012.

STEVENS, A.; SCHMIDT, M. I.; DUNCAN, B. B. Gender inequalities in non communicable disease mortality in Brazil. Ciência \& Saúde Coletiva, Rio de Janeiro (RJ), v. 17, n. 10, p. 2627-2634, out. 2012.

TEIXEIRA, J. B. et al. Doença de Alzheimer: estudo da mortalidade no Brasil, 20002009. Caderno de Saúde Pública, Rio de Janeiro (RJ), v. 31, n. 4, p. 1-12, abr. 2015.
VERAS, R. P. Estratégias para o enfrentamento das doenças crônicas: um modelo em que todos ganham. Revista Brasileira de Geriatria e Gerontologia, Rio de Janeiro (RJ), v. 14, n. 4, p. 779-786, out./dez. 2011.

VILLELA, L. C. M.; GOMES, F. E.; MELÉNDEZ, J. G. V. Tendência da mortalidade por doenças cardiovasculares, isquêmicas do coração e cerebrovasculares. Revista de Enfermagem UFPE on-line, v. 8, n. 9, p. 3134-3141, set. 2014.

WORLD HEALTH ORGANIZATION WHO. Action Plan for the Global Strategy for the Prevention and control of Noncommunicable Diseases. Geneva: WHO, 2008. Disponível em: <http://www.who.int/nmh/ Actionplan-PC-NCD-2008.pdf>. Acesso em: 23 nov. 2012.

. Global status report on noncommunicable disease. Geneva: WHO, 2014. . Noncommunicable Diseases Contry

Profiles 2011. Geneva: WHO, 2011. 